Intersections

Canadian Journal of Music

Revue canadienne de musique
Intersections CANADIAN JOURAL OF MUSIC

\title{
Lévi-Strauss, homme de sens et de paradoxe
}

\section{Monique Desroches}

Volume 30, numéro 1, 2010

URI : https://id.erudit.org/iderudit/1003499ar

DOI : https://doi.org/10.7202/1003499ar

Aller au sommaire du numéro

\section{Éditeur(s)}

Canadian University Music Society / Société de musique des universités canadiennes

ISSN

1911-0146 (imprimé)

1918-512X (numérique)

Découvrir la revue

Citer cet article

Desroches, M. (2010). Lévi-Strauss, homme de sens et de paradoxe.

Intersections, 30(1), 65-75. https://doi.org/10.7202/1003499ar

\section{Résumé de l'article}

L'auteure de cet article lance, sous la lorgnette de l'ethnomusicologue, des pistes de réflexion autour de l'oeuvre de ce grand maître et soulève quelques interrogations relatives à ses positions méthodologiques ainsi qu'à sa conception de l'analyse ethnographique. Sans remettre en cause la fécondité et la richesse des propositions de l'anthropologue français, l'article met en exergue certaines positions de Lévi-Strauss qui mériteraient d'être revisitées, à tout le moins, relativisées si on veut les accorder avec les principes de l'ethnomusicologie contemporaine. L'auteure se concentre à cette fin sur trois aspects de la pensée de Lévi-Strauss : la place du terrain, la notion du temps et celle du changement.
All Rights Reserved (C Canadian University Music Society / Société de musique des universités canadiennes, 2011
Ce document est protégé par la loi sur le droit d'auteur. L'utilisation des services d'Érudit (y compris la reproduction) est assujettie à sa politique d'utilisation que vous pouvez consulter en ligne.

https://apropos.erudit.org/fr/usagers/politique-dutilisation/ 


\title{
LÉVI-STRAUSS, HOMME DE SENS ET DE PARADOXE
}

\author{
Monique Desroches
}

\section{INTRODUCTION $^{1}$}

L’ethnologie de Lévi-Strauss est bien particulière en ce qu'elle interpelle non seulement les fondements de cette discipline mais aussi ceux de nombreuses autres. Pour plusieurs chercheurs, l'ethnologie de Lévi-Strauss se situe quelque part entre la psychanalyse, la philosophie, la linguistique, la sémiologie, la psychologie cognitive, et l'histoire de l'art. Son regard sur la «diversité du monde» émane dans un premier temps de son intérêt pour la philosophie. S'ouvrant un jour sur ce point à son maître Alain Cresson, il eût avec ce dernier un échange verbal qui fut déterminant pour sa carrière. Plutôt que de l'encourager à continuer dans la voie philosophique, Cresson prononce une phrase qui va marquer la démarche du futur chercheur : "vous n'êtes pas fait pour la philosophie, ditil, mais plutôt pour quelque chose d'à côté» (Bertholet 2003, p. 29). Ce "quelque chose d'à côté» s'est avéré être l'ethnologie, discipline qui va le rapprocher du monde des objets, Lévi-Strauss ne voulant pas se limiter aux investigations abstraites de la philosophie. Toutefois, on verra combien l'ethnologie qu'il développe est basée sur peu d'expériences de terrains. Intrigué par les modes de pensée «sauvage», par les mythes et les structures, il portera davantage son regard sur la pensée humaine que sur les aspects superficiels du réel. Dans sa démarche, il constate rapidement que tout semble donné, mais rien ne l'est dans les faits. Aussi, il lui apparaît évident que "pour entrer dans le monde, il faut accepter de passer par des médiations et des procédures» (Bertholet 2003, p. 27). Cet énoncé résume assez bien le cœur de la pensée lévi-straussienne, une pensée autour de laquelle ne convergent pas nécessairement tous les chercheurs. Mais n'est-ce pas là aussi la marque d'une grande œuvre, celle d'inciter à la réflexion et à une incessante remise en question des acquis?

Moult ouvrages ont été consacrés à Lévi-Strauss et le centenaire de sa naissance, particulièrement, a suscité un regain d'intérêt pour son œuvre. L'événement qui nous a réunis (table-ronde) était une manifestation de cet intérêt. Ma participation à celui-ci supposait dans un premier temps un retour intensif aux sources, à celles de l'écrivain, du philosophe et de l'ethnologue qui m’avait fortement marquée comme étudiante dans les années 1970, alors que

1 Je tiens à remercier mes collègues Nathalie Fernando et Jean-Jacques Nattiez pour avoir organisé cette table-ronde en novembre 2008 sur ce penseur du siècle qu'est Lévi-Strauss, et ce, à partir du récent ouvrage de J.-J. Nattiez, «Lévi-Strauss musicien». J’ai eu grand plaisir à lire ce livre dans un premier temps, à l'étape du manuscrit, puis à le relire dans sa forme éditée. 
le structuralisme était à son zénith. La relecture de ses travaux a provoqué un plaisir renouvelé mais également fait naître une série de questions que je souhaite ici partager.

D’emblée, je soulignerai que cet article ne se veut pas une critique systématique du structuralisme lévi-straussien, ce que plusieurs ont déjà fait. Les lignes qui suivent lancent plutôt, sous la lorgnette de l'ethnomusicologue, des pistes de réflexion autour de l'œuvre de ce grand maître et soulèvent quelques interrogations relatives à ses positions méthodologiques ainsi qu'à sa conception de l'analyse ethnographique. Lévi-Strauss ne serait sans doute pas étonné de ce regard critique. Dans un entretien qu'il accordait en 1988 à la radio française «France Inter», il affirmait au journaliste Jacques Chancel qu' «une fois écrits, ses livres lui deviennent étrangers» (Chancel 1988). Voulait-il signifier par là le difficile arrimage de la pérennité de l'écrit avec l'évolution incessante de la pensée scientifique? Ce problème d'actualisation des écrits du chercheur est aussi soulevé par son biographe Bertholet qui écrivait que

vingt ans après la parution de l'Anthropologie structurale, Lévi-Strauss ne se reconnaîtra pas entièrement dans son livre. Cette œuvre, dira t-il, contient « des développements qui me font aujourd'hui frémir tant ils sont trop simples, trop schématiques ... Un livre, c'est du travail passé». (2008, p. 232-233)

À ce premier problème s'ajoute celui de l'interprétation de ses écrits par le lecteur dont Lévi-Strauss redoute le jugement posthume. Il précise un peu plus loin dans cet entretien avec Chancel : «je ne suis pas convaincu que ce que l'on retiendra de moi, correspondra à ce que j'aurais souhaité que l'on retienne vraiment de ma pensée et que je juge important» (Chancel 1988).

Je mavancerai donc ici avec grande prudence, étant bien consciente que la lecture que je fais de son œuvre est imprégnée de mes a priori méthodologiques, mais surtout, marquée par mes expériences singulières de terrains ethnomusicologiques. Sans remettre en cause la fécondité et la richesse des propositions de l'anthropologue français, je mettrai en exergue certaines de ses opinions qui mériteraient à mon sens d'être relativisées, notamment quand il s'agit de les accorder avec les principes de l'ethnomusicologie contemporaine. Plus précisément, je me concentrerai sur trois aspects de la pensée de Lévi-Strauss : la place du terrain, la notion du temps et celle du changement.

\section{LÉVI-STRAUSS ET LE TERRAIN}

L'ensemble de l'œuvre de Lévi-Strauss pourrait être résumé dans cette prémisse : la pensée sauvage est rationnelle, organisée, scientifique. La Pensée sauvage (1962) montre, par exemple, la logique rigoureuse sous-jacente au totémisme. Toutefois, il importe de souligner que ses écrits émanent moins de données issues de ses propres terrains que de la synthèse de recherches menées par d'autres ${ }^{2}$. Tristes tropiques (1955) est un des rares ouvrages où l'on retrouve des

2 Tristes tropiques demeure un de ses rares ouvrages émanant directement des observations de terrain et le «je» se retrouve au cœur du récit. 
données personnelles de terrain, sans que celles-ci ne soient toutefois consignées selon les normes habituelles de l'ethnographie. Mais même si le chercheur s'est peu adonné à l'observation in situ, ce livre a marqué le champ de l'ethnographie en ouvrant la voie à une nouvelle méthode, celle du sujet observant, avec ses émotions, ses sentiments qui se confondent là avec les consignations objectives et rationnelles du chercheur. Cette singularité a d'ailleurs fait l'objet de critiques, certains voyant le livre davantage comme un récit de voyage qu'un essai scientifique, qualifiant même l'ensemble de son œuvre de philosophie empirique.

Dans plusieurs de ses écrits, dont Le cru et le cuit, Lévi-Strauss (1964) remet en question la valeur de l'enquête in situ, celle aussi de l'observation participante car il en voit vite les limites. C'est le cas de l'Introduction à l'anthropologie structurale où il reconnaît que : «la connaissance dérive directement du contact avec le terrain [...] mais le manifeste n'est jamais que le vernis et la clé de l'explication se trouve toujours dans le latent, i.e., le caché, et c'est là précisément la tâche de l'ethnologue de la retrouver" (dans Deliège 2001, p. 38). Cherchant à comprendre le champ de l'articulation nature/culture et surtout le mode de "pensée sauvage", Lévi-Strauss se concentrera toute sa vie à la compréhension de la complexité humaine, et à la mise en perspective de cette pensée sauvage qu'il considère procéder de la même logique que la pensée scientifique (1962). Chez Lévi-Strauss, la structure est une donnée inconsciente qui doit être révélée par l'analyse. L'observation n'est qu'illusion et c'est pourquoi il importe d'aller au-delà de ce premier état de conscience, c'est-à-dire, de le transcender. Partant de cette conception, on comprend mieux le peu d'importance que Lévi-Strauss accorde au terrain, voire aux discours autochtones. Critiquant à ce propos la place que réserve le chercheur à la parole du terrain, Deliège souligne certains énoncés du Cru et le cuit (1964), tels que «les indigènes répondent qu'ils ne savent rien ou que leurs ancêtres ont toujours vécu de la sorte, [...] ils sont incapables d'une explication satisfaisante» (2001, p. 39). Ces propos, s'ils illustrent bien le rôle complémentaire de l'analyse dans la quête de connaissance d'une culture, sont néanmoins étonnants et paradoxaux si on remonte à la pensée du jeune chercheur désireux d'ancrer ses investigations philosophiques dans un monde concret. La rigueur méthodologique concernerait-elle plus spécifiquement pour lui le niveau de l'analyse, le terrain, non concerné par cette étape de recherche, se réduisant vraisemblablement à une étape de collecte de matériau brut? Pourtant, les choix de collecte et les sélections du regard relèvent bien eux aussi de l'analyse. Les observations effectuées sur le terrain ne peuvent être totalement neutres, étant notamment ancrées dans des a priori théoriques et des objectifs de recherche qui animent le chercheur sur le terrain. De plus, le terrain est-il si muet sur sa propre culture, ainsi que le prétend Lévi-Strauss? L'indicibilité relève-t-elle vraiment d'une incapacité de l'indigène à l'abstraction? Il est permis d'en douter, d'autant plus que dans La pensée sauvage, Lévi-Strauss (1962) met justement de l'avant la dimension scientifique des cultures de l'oralité, notamment par une habileté à procéder à des classifications rigoureuses. La connaissance de l'Autre se révèle non seulement par l'analyse comme le montre bien Lévi-Strauss dans son œuvre, 
mais aussi, «dans » et "par» le discours du terrain, approche trop peu explorée par le maître. Plus encore, l'anthropologie contemporaine, celle notamment de Geertz (1973), et de Clifford et Marcus (1986) prône la mise en place d'un dialogue entre les deux mondes, celui du chercheur et celui du milieu local, une approche qui a porté fruit en ethnomusicologie. Et pour illustrer la valeur irremplaçable de cette rencontre interne/externe et surtout de la pertinence des connaissances locales dans l'édification du système symbolique, je me référerai maintenant à une recherche sur une musique rituelle d'ascendance tamoule à la Martinique (Antilles françaises), recherche démarrée en 1979 et qui se poursuit.

\section{DE L'IMPORTANCE DU DIALOGUE INTERNE/EXTERNE}

Cherchant à connaître les règles de fonctionnement de battements de tambours qui accompagnaient une cérémonie rituelle d'origine tamoule, et tentant surtout de mettre en évidence le mode de fonctionnement symbolique entre la musique et le rituel, j'ai effectué une série d'opérations sur le terrain allant de la présence systématique aux cérémonies rituelles, à la notation des éléments du déroulement rituel, aux entrevues auprès de musiciens, à la transcription des données enregistrées, à des expérimentations et à des retours sur le terrain pour validation d'hypothèses. Je présenterai ici deux de ces étapes. La première renvoie à ce que l'on pourrait qualifier d'expérimentation sur le terrain, et la deuxième concerne davantage une étape de laboratoire, une analyse externe menée exclusivement par le chercheur en l'absence des autochtones.

Lors d'une séance de travail sur le terrain, j'ai réuni autour d'un montage sonore, rassemblant les huit battements de tambour différents réalisés au cours des cérémonies, certains informateurs (musiciens et officiants du culte). Cherchant à saisir les modes d'arrimage entre la musique et les phases cérémonielles, je leur ai demandé d'identifier le battement de tambour qui leur était présenté et de l'associer selon leur connaissance à la phase cérémonielle que ce battement accompagnait. Répondant à la question : «de quel battement de tambour s'agit-il?», certains informateurs précisent alors non seulement l'étape cérémonielle qui lui est associée, mais donnent une information qui renvoie à un autre niveau, celle du nom d'une divinité. Je présenterai maintenant de façon schématique les tableaux issus de cette recherche. Bien que déjà publiés (Desroches 1996), il me semble utile de les mobiliser ici afin d'illustrer la complémentarité des regards internes-externes, et surtout de montrer la pertinence des retours successifs sur le terrain. Extraits de cette publication, les deux prochains tableaux visent à mettre en exergue la valeur irremplaçable de l'étape de confrontation des hypothèses de recherche avec les locaux. Le tableau 1 résume d'abord les réponses obtenues suite à une présentation-synthèse (montage sonore) des battements rituels. 
Tableau 1

\begin{tabular}{|c|c|c|c|c|}
\hline \multicolumn{5}{|c|}{ Expérimentation sur le terrain } \\
\hline PHASE DE LA CÉRÉMONIE & $\begin{array}{l}\text { BATTEMENTS DE } \\
\text { TAMBOUR }\end{array}$ & \begin{tabular}{|c|} 
Premier \\
classement
\end{tabular} & $\begin{array}{l}\text { RÉPONSES LIVRÉES SUR LE } \\
\text { TERRAIN }\end{array}$ & \begin{tabular}{|c|}
$\begin{array}{c}\text { Classement } \\
\text { culturel }\end{array}$ \\
\end{tabular} \\
\hline $\begin{array}{l}\text { Purification et offrande d'aliments } \\
\text { végétariens offerts aux divinités } \\
\text { (Préparation) }\end{array}$ & 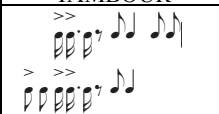 & $\begin{array}{l}\text { B-1 } \\
\text { B-1' }\end{array}$ & Rassemblement et appel à Maliémin. & $\begin{array}{l}\text { B-1 } \\
\text { B-1' }\end{array}$ \\
\hline $\begin{array}{l}\text { Offrande des aliments. } \\
\text { Quand on dépose le plateau par terre. } \\
\text { Avant d'immoler les animaux. } \\
\text { L'élévation du mât. }\end{array}$ & $\dot{\rho} \dot{\rho} \dot{\rho} \dot{\rho}$ etc... & B-2 & Purification et offrandes d'aliments. & B-2 \\
\hline $\begin{array}{l}\text { (Suivant l'invocation à Maliémin) } \\
\text { Cuisson du riz au lait. }\end{array}$ & 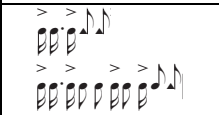 & $\begin{array}{l}\text { B-3 } \\
\text { B-3' }\end{array}$ & $\begin{array}{l}\text { Appel à Maliémin. } \\
\text { Cuisson du riz au lait. }\end{array}$ & $\begin{array}{l}\text { B-1" } \\
\text { B-1"' }\end{array}$ \\
\hline Intervient à différents moments. & 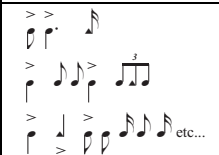 & B-4 & $\begin{array}{l}\text { Battement pour accorder les tambours } \\
\text { (demandé par le chef-tambourineur). }\end{array}$ & B-3 \\
\hline Transe du prêtre. & 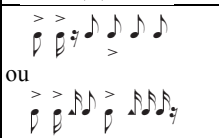 & B-5 & Appel à Maldévilin. & B-4 \\
\hline Communion & 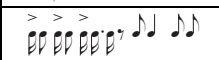 & B-6 & Appel à Maliémin. & B-1"'" \\
\hline $\begin{array}{l}\text { Après le sacrifice des animaux on se } \\
\text { dirige vers la maison des célébrants. }\end{array}$ & 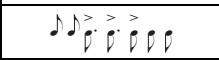 & B-7 & Appel pour toutes les divinités. & B-5 \\
\hline
\end{tabular}

Transcription: M.Desroches

Tableau: D. Amorocho

Trois battements $(1,3$ et 6$)$ sont associés à l'appel à Maliémin, tandis que le $5^{\mathrm{e}}$ battement est selon ces musiciens destiné à la divinité Maldévilin. Un premier problème émane de cette expérience de terrain : les battements 1,3 et 6 , malgré leurs profils rythmiques distincts, sont associés par les répondants, à une seule divinité : Maliémin. J'étais alors confrontée à une ambiguïté : comment des battements de tambour distincts pouvaient-ils être associés à différentes étapes cérémonielles, tout en gardant leur référence à une seule et même divinité? Comment aussi s'articulaient les liens de fonctionnalité symbolique entre la musique, le déroulement cérémoniel et l'appel des divinités dans cette apparente incohérence? Ces données émiques (internes à la culture) m’ont obligée à revenir à une étape de laboratoire, i.e. à l'analyse externe.

C'est là où les propositions de Lévi-Strauss se sont avérées non seulement pertinentes, mais fécondes. Il faut se rappeler que selon ce dernier, la culture commence avec la règle. C'est elle qui institue un ordre. À ses yeux, le réel forme un ensemble désordonné qui a besoin d'être pris en charge par l'esprit humain, avant de faire sens. Le rôle du chercheur est alors de mettre en exergue par le truchement de l'analyse, ce sens non révélé.

Dans le déroulement de cette recherche, la mise en paradigme (tableau 2 ci-dessous) est venue apporter des éléments intéressants de connaissance systémique. 
Cette mise en paradigme révèle des liens de parenté structurelle entre les battements 1,3 et 6 , puisque ces trois éléments comportent une même cellule rythmique de trois notes, cellule qui a permis d'élaborer une hypothèse autour du processus d'attribution symbolique : la référence à la divinité Maliémin pourrait émaner de la présence de cette seule cellule tripartite. Cette dernière constituerait alors une unité minimale de signification extra-musicale (ici, Maliémin) qui peut s'apparenter à une "matrice» ou à la notion de "modèle » chez Lévi-Strauss. Ce tableau illustre aussi la présence d'une musique structurée et ordonnée qui donne sens à la cérémonie rituelle, et ce sens n’a pu être

Tableau 2

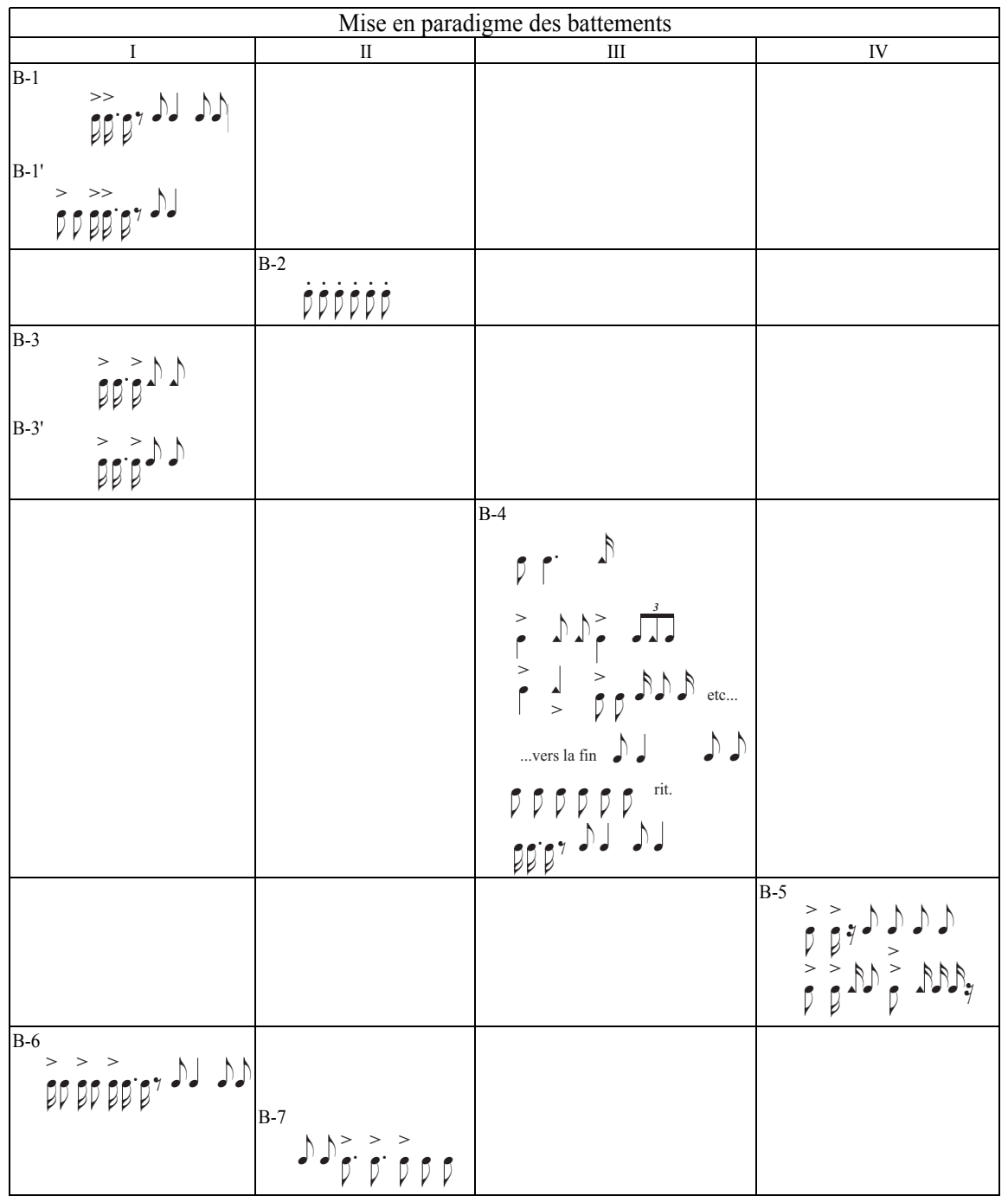

Transcription: M.Desroches

Tableau: D. Amorocho 
révélé que par cette étape d'analyse externe. À ce niveau, Lévi-Strauss avait bien raison d'insister sur le rôle de l'analyste (pour lui, l'ethnologue) comme révélateur de sens caché. De plus, des retours sur le terrain ont permis de valider la pertinence culturelle de cette analyse externe. Cette étape de retour sur le terrain pour validation d'hypothèses semble toutefois étrangère à LéviStrauss. À ce chapitre, il apparaît paradoxal que pour un chercheur aussi préoccupé de rigueur et de méthode, il ne pose pas la question de la pertinence culturelle des analyses, celle qui passe non seulement par des observations ethnographiques rigoureuses, mais aussi par des retours sur le terrain et des ajustements d'hypothèses de recherche avec les autochtones. Le terrain, voire les allers-retours entre ce dernier et le laboratoire font également partie intégrante de l'arsenal scientifique.

\section{STRUCTUREL ET/OU STRUCTURANT ?}

Lévi-Strauss appelle "sens» l'arrangement des éléments dans un ensemble considéré (liens de parenté, groupe de mythes). Auquel cas, le travail d'interprétation est achevé lorsqu'on a réussi à mettre en évidence les dispositifs de relations gouvernant ces éléments. Dans cette foulée, et ainsi que souligné par Hénaff, le sens ne serait rien d'autre que la structure manifestée (Hénaff 2008, p. 64). Poussons plus loin cette conception et examinons son incidence sur l'analyse ethnomusicologique. Car le sens musical n'est-il que structurel ou structure révélée par l'analyse? Ne peut-il être aussi structurant, c'est-à-dire, un acteur fort dans la structuration d'une société en train de se construire ou de se reconstruire? On atteint là une autre conception du terme structure qui nécessite, pour être prise en charge, une inscription ou un ancrage fort du chercheur sur le terrain. Cette approche se profile dans le courant du structuralisme empirique tel que prôné par Radcliffe-Brown, un chercheur avec qui Lévi-Strauss a eu, et ce, dans un respect mutuel, des échanges polémiques. Pour Lévi-Strauss, il faut se rappeler que le mythe est une réalité en dehors du temps. Le mythe constitue une sorte de réalité autonome qu'il importe d'étudier en elle-même et pour ellemême sans référence à un contexte. Toutefois, même si son essence se profile en dehors du temps, sa réalisation ou sa performance est quant à elle, bien implantée dans le concret, dans un temps historique et social. À l'instar des rituels, la performance ne cherche pas qu'à exprimer une idée, mais elle cherche aussi à maintenir ou à modifier le cours des choses dans le social immédiat, comme le dit à juste titre Marcel Hénaff (2008). C’est pourquoi, pour Radcliffe-Brown, la structure renvoie à un ensemble de relations unissant les individus d'un groupe social. Plus précisément, elle traduit la manière dont les membres d'une société donnée s'organisent entre eux. Elle est agissante, opérante, consciente. Dès lors, le problème n'est plus de savoir ce que les symboles ou plutôt les dispositifs symboliques signifient, mais plutôt de comprendre comment ils fonctionnent dans la société : quel monde ou quelle société est-on en train de construire ou de reconstruire? (Hénaff 2008, p. 115)

Acquiesçant à cette posture théorique, il m’apparaît qu'un service rituel ne met pas en place qu'un texte et qu'une musique dans un contexte donné. 
"Quelque chose se passe» lors de la performance. Celle-ci engendre un cotexte (Desroches 2009), c'est-à-dire un espace né de la rencontre du texte musical, de l'espace rituel et de l'espace social. Mettre en acte un rituel est beaucoup plus que la perpétuation d'un contenu religieux, mythique : c'est la réactualisation d'une société que l'on construit, consolide, dé-construit ou re-construit. Ainsi, le chercheur est certes un passeur de sens, comme le rappelle si bien Lévi-Strauss. Dans les faits, les deux hommes (Lévi-Strauss et Radcliffe-Brown) cherchent à dire le sens, à le révéler. Toutefois, dans un cas, celui de Lévi-Strauss, le sens est dans l'œuvre, dans les mythes par exemple, alors que chez Radcliffe-Brown, il est dans le social et ses modalités de réalisation. Pour illustrer la fécondité de cette dernière posture théorique proposée par Radcliffe-Brown, je m'appuierai maintenant sur une série de terrains effectués depuis 1987 sur les musiques rituelles pratiquées par les descendants tamouls à l'île de la Réunion dans l'océan Indien.

\section{ObSERVER LE CHANGEMENT}

Amenée depuis 1987 à suivre le groupe indo-créole d'ascendance tamoule à la Réunion, j'ai pu observer des modifications profondes au chapitre des musiques et des espaces rituels. Issu d'une Inde villageoise de basse caste, le groupe s'est installé dans l'île au milieu du XIX ${ }^{\mathrm{e}}$ siècle. Il s'est par les années scindé conséquemment aux changements de statut socioéconomique qu'il a connu. Une partie de ce groupe initial est en effet demeurée active dans le travail agricole au sein des plantations, en milieu rural, alors qu'une autre a migré vers la ville, migration qui s'est accompagnée pour bon nombre d'entre eux, d'un rehaussement du statut social et économique. Or, dans le monde indien, statut social, religion et musique sont étroitement imbriqués. Les pratiques musicales et religieuses apparaissent alors comme des vecteurs privilégiés pour marquer clairement les différences sociales. Elles participent ici à l'édification de deux ensembles religieux et musicaux cohérents en réponse, voire en harmonie avec les statuts sociaux. De plus, comme l'indique le tableau 3 ci-dessous, ce ne sont pas que les structures formelles des répertoires qui changent selon le contexte : ce sont aussi les modalités de transmission, les procédés stylistiques, les finalités visées par chacun des deux rituels participant ainsi à l'édification d'une société en harmonie avec ces valeurs religieuses et musicales.

L'ensemble de ces éléments constitue un tout performant et cohérent, et procure une signature identitaire à chacun des groupes utilisateurs. À la lecture de ce tableau, on réalise combien il importe de se pencher sur l'ensemble des pratiques distinctives de chacun des groupes pour saisir le sens non seulement musical et religieux, mais aussi social. Les structures rituelles et musicales ici révélées résultent de réseaux sociaux qui se construisent selon les valeurs et croyances adoptées. Le jour où, par exemple, on observera la présence d'un harmonium, instrument de prédilection pour les cérémonies de louange en milieu urbain au sein de l'ensemble musical des rituels sacrificiels de plantation, ce sera là le présage d'un changement important de paradigme esthétique et stylistique dans les traditions musicales et religieuses. 
Tableau 3

\begin{tabular}{||l|l||}
\hline \multicolumn{1}{|c|}{ Musique rituelle de sacrifice animal } & \multicolumn{1}{c|}{ Musique de cérémonie de louange } \\
\hline Homophonie (battements de tambour) & $\begin{array}{l}\text { Hétérophonie (tablas, voix, mridangam, } \\
\text { harmonium) }\end{array}$ \\
\hline $\begin{array}{l}\text { Emphase sur la répétition de patrons } \\
\text { rythmiques }\end{array}$ & Place importante accordée à l'improvisation \\
\hline $\begin{array}{l}\text { Les tambourineurs doivent jouer ensemble le } \\
\text { même rythme (conception verticale) }\end{array}$ & $\begin{array}{l}\text { Développement linéaire des patrons rythmiques } \\
\text { et mélodiques }\end{array}$ \\
\hline Apprentissage : patrilinéarité & Apprentissage : cours (maître/disciple) \\
\hline $\begin{array}{l}\text { Fonction principale : invoquer les divinités, } \\
\text { posséder l'officiant du culte lors de la transe } \\
\text { (musique fonctionnelle) }\end{array}$ & $\begin{array}{l}\text { Fonction principale : honorer les divinités, saluer } \\
\text { leur présence (musique d'accompagnement) }\end{array}$ \\
\hline $\begin{array}{l}\text { Musique vue comme médiation entre le monde } \\
\text { des dieux et celui des hommes }\end{array}$ & $\begin{array}{l}\text { Musique vue comme espace de création } \\
\text { artistique }\end{array}$ \\
\hline $\begin{array}{l}\text { Geste musical : perpétuation d'une mémoire } \\
\text { collective }\end{array}$ & $\begin{array}{l}\text { Geste musical : mise en valeur du talent } \\
\text { individuel }\end{array}$ \\
\hline
\end{tabular}

En plaçant cette dernière approche dans la foulée des propositions de LéviStrauss, une caractéristique émerge : on réalise combien cette démarche est axée sur la mise en acte de l'œuvre, c'est-à-dire sur la pratique musicale rituelle vue comme pratique sociale, et non seulement sur la musique vue comme objet. En effet, le choix de tel instrument de musique dans un contexte rituel spécifique suppose une série de gestes musicaux dictés par une philosophie religieuse et musicale, des gestes qui structurent socialement à leur tour le groupe utilisateur. Un regard sur le seul objet, sans cette mise en relation entre contexte et sujet musicien, n'aurait pu révéler l'enjeu social de la performance. Ce changement de perspective aurait sans doute permis à Lévi-Strauss de relativiser son regard sur l'art moderne avec lequel il a entretenu des liens ambigus, faute de le comprendre. «Cette forme artistique a dissous l'objet, écrit-il dans le 'Regard éloigné' (1983), et par là perdu le monde [...] L’impressionnisme avait ouvert cette voie funeste, poursuit-il, en tentant d'illustrer ce principe que peindre, c'est traduire des moments de la subjectivité percevante" (op.cit., p. 339). Pourtant, dans son analyse des mythes, le rôle de l'analyste est clair, ainsi que le rappelle Hénaff : «un musicien lit une partition et en reprend l'exécution; il ne s'agit pas de dégager une signification mais de constater une opération. Son rôle n'est pas de détecter le sens des récits (comme on le ferait pour des signes). Il s'agit de comprendre en quoi ils ordonnent un monde où la vie aura un sens » (op.cit., p. 190). Avec cette conception de la création musicale, il est permis de se demander comment il aurait procédé pour décrypter et comprendre des cultures musicales comme celles de l'Inde ou celle du jazz qui se caractérisent par la signature stylistique de l'interprète, par l'improvisation. Les procédés performanciels sont au cœur de la création musicale : les pratiques constituent là des œuvres ouvertes où l'interprète crée, in situ, SON œuvre. 


\section{L'EFFET DU TEMPS}

Lévi-Strauss est l'ethnologue de la continuité. Dans cette foulée, il est tout à fait concevable, et même logique, qu'il soit contre les Modernes et qu'il comprenne difficilement la musique contemporaine qu'il trouve an-émotive. La temporalité musicale devait bien le déranger, le mythe recouvrant, comme souligné antérieurement, un caractère atemporel. En fait, il serait intéressant de creuser chez lui cette notion temporelle, ce rapport au temps. La discontinuité et le changement social lui sont pénibles. Il le dit explicitement dans Tristes tropiques quand, face à la modernité qu'il observe dans les îles, il passe de l'émerveillement au regret. Tout se passe comme si Lévi-Strauss, en tentant de comprendre les modes de pensée sauvage, évacuait toute forme de changement au profit de la mise en exergue d'une pérennité culturelle et sociale, une conception qui me semble aller à l'encontre de l'esprit d'adaptation des sociétés primitives que l'ethnologue cherche pourtant à comprendre. En se concentrant sur la structure, l'ethnologue serait-il passé à côté des enjeux et du processus de construction de l'objet?

\section{Conclusion}

Certains aspects de la pensée de ce grand chercheur enchantent, d'autres appellent au débat. Je retiendrai de l'œuvre la minutie de ses analyses, l'originalité de ses propositions, la rigueur des démonstrations, l'importance de dégager des règles et des modèles générateurs. L'admiration que son œuvre a suscitée n'empêche pas toutefois la critique de sa démarche. Au plan ethnomusicologique par exemple, une transposition directe de ses propositions aurait notamment gommé le sujet musicant, la singularité des performances, le rôle actif de l'écoute, du sensible, une démarche qu'a notamment bien menée Feld dans son ouvrage Sound and Sentiment (1982). Il aurait aussi été intéressant de voir œuvrer Lévi-Strauss en dehors de sociétés primitives, au bénéfice d'enquêtes en milieu urbain et multiethnique, un espace pas forcément organisé selon les mêmes règles des sociétés dites primitives, là où le don et l'échange sont des activités structurantes et où se jouent de constantes négociations identitaires. Mais Lévi-Strauss aurait-il été tenté par ce voyage dans ce monde à la pensée moins «sauvage»?

Quoiqu'il en soit, l'œuvre de Lévi-Strauss demeure une source inépuisable d'inspiration. Même si Lévi-Strauss ne cherchait ni le succès, ni la reconnaissance, l'ensemble de la communauté scientifique s'accorde aujourd'hui pour reconnaître l'apport magistral de l'œuvre du chercheur et du penseur. Ses écrits ont non seulement nourri la trajectoire de nombreux scientifiques, mais ont suscité un large débat dans la communauté des chercheurs. Il aura ainsi fait progresser les disciplines des sciences humaines.

\section{RÉFÉRENCES}

Bertholet, Denis. 2003. Claude Lévi-Strauss. Plon, Paris.

Chancel, Jacques 1988. Claude Lévi-Strauss, Radioscopie. CD d'émission radiophonique, Paris : France Inter, INA. 
Clifford, James et George E Marcus. 1986. Writing Culture. Berkeley : University of California Press.

Deliège, Robert. 2001. Introduction à l'anthropologie structurale : Lévi-Strauss. Paris : Seuil.

Desroches, Monique. 1996. Tambours des dieux. Montréal et Paris : L'Harmattan. . 2009. «Entre texte et performance : l'art de raconter». Cahiers d'ethnomusicologie, $\mathrm{n}^{\mathrm{O}}$ 21, Genève : 103-115.

Feld, Steven. 1982. Sound and Sentiment : Birds, Weeeping, Poetics and Song in Kaluli Expression. Philadelphie : University of Pennsylvania Press.

Geertz, Clifford. 1973. The interpretation of culture. New-York : Basic.

Hénaff, Marcel. 2008. Claude Lévi-Strauss, le passeur de sens. Paris : Perrin.

Joulia, Émilie. 2008. Lévi-Strauss. L'homme derrière l'œuvre. Paris : J.C. Lattès.

Lévi-Strauss, Claude. 1955. Tristes tropiques. Paris : Plon. . 1958 (1974). Anthropologie structurale. Paris : Plon. 1962. La pensée sauvage. Paris : Plon.

1964. Mythologiques, Le cru et le cuit. Paris : Plon. 1983. Le regard éloigné. Paris : Plon.

Nattiez, Jean-Jacques. 2008. Lévi-Strauss musicien. Essai sur la tentation homologique. Arles : Actes Sud.

\section{RÉSUMÉ}

L'auteure de cet article lance, sous la lorgnette de l'ethnomusicologue, des pistes de réflexion autour de l'œuvre de ce grand maître et soulève quelques interrogations relatives à ses positions méthodologiques ainsi qu'à sa conception de l'analyse ethnographique. Sans remettre en cause la fécondité et la richesse des propositions de l'anthropologue français, l'article met en exergue certaines positions de Lévi-Strauss qui mériteraient d'être revisitées, à tout le moins, relativisées si on veut les accorder avec les principes de l'ethnomusicologie contemporaine. L'auteure se concentre à cette fin sur trois aspects de la pensée de Lévi-Strauss : la place du terrain, la notion du temps et celle du changement.

\section{ABSTRACT}

Through the lens of the ethnomusicologist, the author of this article provides food for thought about the work of this great master and raises some questions about his methodological position and his conception of ethnographic analysis. Without questioning the fertility and richness of the French anthropologist's proposals, the article highlights some of Lévi-Strauss's positions that deserve to be revisited and, at the very least, qualified if we want them to accord with the principles of contemporary ethnomusicology. The author focuses to this end on three aspects of the thought of Lévi-Strauss: the importance of place, and the concepts of time and change. 